\title{
Consciência morfossintática, alfabetização e contextos do desenvolvimento
}

\author{
Márcia da Mota - Universidade Federal de Juiz de Fora, Juiz de Fora, Brasil \\ Daniela Cristina Belchior Mota - Universidade Federal de Juiz de Fora, Juiz de Fora, Brasil \\ Junia Cota - Universidade Federal de Juiz de Fora, Juiz de Fora, Brasil \\ Stella Mansur - Universidade Federal de Juiz de Fora, Juiz de Fora, Brasil \\ Simone Lima - Universidade Federal de Juiz de Fora, Juiz de Fora, Brasil \\ Atila Calzavara - Universidade Federal de Juiz de Fora, Juiz de Fora, Brasil \\ Natália Cunha - Universidade Federal de Juiz de Fora, Juiz de Fora, Brasil \\ Eliane Banhato - Universidade Federal de Juiz de Fora, Juiz de Fora, Brasil
}

\begin{abstract}
Resumo
Este artigo apresenta dados sobre um estudo que investigou o papel da escolarização sobre o desenvolvimento da consciência metassintática. Cinquenta e quatro crianças oriundas da primeira e segunda séries de escolas privadas e estaduais participaram deste estudo. A elas foram dados três tarefas de consciência sintática: julgamento de sentenças corretas, julgamento de sentenças incorretas e correção. Não foi encontrados um efeito estatisticamente significativo para série, mas se encontrou um efeito significativo para o tipo de escola. Crianças de escolas particulares tiveram um desempenho melhor do que crianças de escolas públicas. Correlações entre as medidas de consciência sintática mostraram que quanto melhor as crianças eram medidas em consciência sintática, melhor elas eram nas tarefas de escrita. Os resultados sugerem que a experiência das crianças no seu meio ambiente tem um papel no desenvolvimento da consciência sintática.

Palavras-chave: alfabetização; consciência sintática; desenvolvimento metalinguístico.
\end{abstract}

\section{Morpho-syntatic awareness, literacy and developmental contexts}

\begin{abstract}
This paper reports data of a study that investigated the role of schooling in metasyntactic awareness development. Fifty-four children drawn from first and second grade from private and state schools participated in the study. They were given three syntactic awareness tasks: judgement of correct and incorrect sentences and correction. There were no grade-effect but a significant school effect. Children from private schools performed better than from public schools. Correlations between syntactic awareness and literacy measures showed that the better children were at syntactic awareness the better they were at literacy measures. The results suggest that children's environmental experience have a role to play in the development of syntactic awareness.

Keywords: literacy; syntactic awareness development; metalinguist development.
\end{abstract}

\section{Introdução}

A habilidade de refletir sobre a linguagem como objeto do pensamento é chamada de habilidade metalinguística (Garton \& Pratt, 1989). Pesquisadores interessados no desenvolvimento metalinguístico passaram boa parte da década de 80 discutindo questões básicas sobre o seu desenvolvimento: a idade de aquisição, a relação entre esta habilidade e alfabetização e as implicações pedagógicas dos resultados de pesquisa.

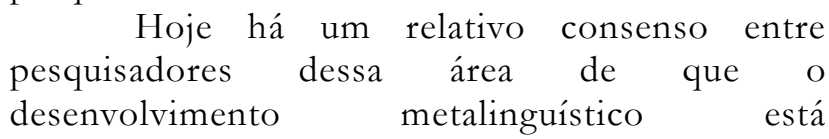

${ }^{1}$ Endereço para correspondência:

Universidade Federal de Juiz de Fora - Instituto de Ciências Humanas - Departamento de Psicologia - Campus Universitário Martelos - CEP: 36036330

E-mail: mmotapsi@terra.com.br

Protocolo comitê de ética: CEP-HU/UFJF 0581.1.180.000-05 intrinsecamente relacionado com $\mathrm{O}$ desenvolvimento da alfabetização. Algum grau de reflexão sobre a linguagem é necessário para que a criança possa se alfabetizar, mas a habilidade realmente metalinguística é decorrente das aprendizagens explícitas decorrentes da escolarização (Gombert, 2003).

A esse grau inicial, que representa um grau implícito de conhecimento metalinguístico, Gombert (1992) chamou de epilinguístico. A reflexão explícita sobre a linguagem seria a habilidade metalinguística. Embora, haja muita controvérsia a respeito do que pode ser considerado conhecimento explícito ou implícito em psicologia, pesquisadores concordam que uma habilidade para ser definida como metalinguística precisa envolver uma reflexão consciente sobre a linguagem, e que o indivíduo, para exercê-la, deve explicitamente focalizar sua atenção e manipular a linguagem de forma intencional (Bialystok, 1993; Gombert, 1992). 
Dentre as habilidades metalinguísticas duas aparecem frequentemente associadas à aquisição da língua escrita: a consciência fonológica e a consciência sintática. A consciência fonológica é a habilidade de se refletir sobre os sons que compõem a fala (Cardoso-Martins, 1995). O desenvolvimento dessa habilidade tem sido muito estudado, inclusive no contexto do português do Brasil (Barrera \& Maluf, 2003; Bradley \& Bryant, 1985; Capovilla \& Capovilla, 2000; Cardoso-Martins, 1995; Guimarães, 2003; Plaza \& Cohen, 2004; Rego \& Bryant, 1993; Tunmer, 1990). A importância dessa habilidade para o desenvolvimento reside na relação causal que tem com alfabetização. As crianças capazes de refletir sobre os sons que compõem a fala têm maior facilidade de aprender a ler e a escrever, e o treinamento da consciência fonológica ajuda na prevenção e tratamento dos problemas de leitura (Bradley \& Braynt, 1985).

A consciência sintática envolve a habilidade de se refletir, manipular e mostrar controle intencional sobre a sintaxe da língua (Gombert, 1992). Essa habilidade também foi identificada como facilitadora da aprendizagem da língua escrita (Rego \& Bryant, 1993; Plaza \& Cohen, 2003; Tunmer, 1990).

A relação entre a consciência sintática ou consciência gramatical e a leitura ou a escrita é mais controversa do que a relação dessas últimas com a consciência fonológica. Em primeiro lugar, o termo consciência sintática foi usado tanto para tarefas que envolvem aspectos mais gerais da sintaxe da língua, quanto para tarefas que envolvem aspectos morfológicos. Ainda, alguns autores consideram que não se podem separar aspectos sintáticos dos morfológicos, preferindo o termo morfossintaxe ao termo consciência sintática (Correa, no prelo). No nosso caso específico utilizaremos o termo consciência morfossintática, pois nossas tarefas envolvem tanto habilidades sintáticas como morfológicas.

Em segundo lugar, a contribuição específica da consciência sintática para a alfabetização ainda é motivo de debate. Estudos longitudinais mostram uma associação entre a consciência sintática e a alfabetização (Rego \& Bryant, 1993; Plaza \& Cohen, 2003; Tunmer, 1990). No entanto, alguns autores, como Bowey (2005) questionam o resultado desses estudos. Bowey (2005) argumenta que estudos que mostram a contribuição independente da sensibilidade à sintaxe $\mathrm{da}$ língua para alfabetização, em geral, utilizam critérios pouco conservadores de controle de variáveis. Ao se estudar o valor preditivo das habilidades metalinguísticas para leitura é importante que se controle o conhecimento inicial da leitura. Bowey (2005) argumenta que estudos que investigaram a contribuição da consciência sintática para alfabetização frequentemente incluem crianças com conhecimento rudimentar da língua escrita e as tratam como não-leitores, o que para a autora acaba por aumentar artificialmente o efeito da consciência sintática no desempenho da leitura. Usando um critério mais conservador, Bowey (2005) deu a crianças de quatro anos de idade, nãoleitoras uma série de tarefas cognitivas, de processamento fonológico e de julgamento e correção de sentenças. Os resultados mostraram que as tarefas de consciência sintática/morfossintática não contribuíram de forma independente para leitura e escrita, mas as tarefas de consciência fonológica sim.

As crianças no estudo de Bowey (2005) eram muito pequenas. É nossa opinião que, embora a consciência sintática possa não ter o mesmo peso que a consciência fonológica no desenvolvimento da alfabetização, isso não quer dizer que essa contribuição não seja importante. De fato, outros estudos mostram uma conexão entre essa habilidade e a alfabetização.

Plaza e Cohen $(2003,2004)$ investigaram a contribuição da consciência fonológica, consciência sintática e velocidade de nomeação para a aquisição da escrita e leitura de crianças em processo de alfabetização. O estudo de Plaza e Cohen mostra que as medidas de consciência sintática influenciaram as medidas de leitura e escrita mesmo depois de controlarem o efeito das outras duas variáveis.

Embora as crianças nesse estudo fossem mais velhas (6 anos mais ou menos), podemos concluir que a habilidade de se refletir sobre aspectos sintáticos/morfossintáticos contribui, mesmo que não tão fortemente quanto a consciência fonológica, para o sucesso na alfabetização. Provavelmente a habilidade de refletir sobre a sintaxe começa a ser mais efetiva depois que a criança adquire algum conhecimento sobre as regras de correspondência de sua língua, mas ainda assim essa contribuição pode ser observada.

É possível que a consciência sintática e a consciência fonológica afetem a alfabetização de maneiras diferentes. A consciência fonológica contribuiria para o entendimento da correspondência entre letra e som, isto é, para a 
aquisição do princípio alfabético, e a consciência sintática para leitura contextual (Rego \& Bryant, 1993).

Em um estudo longitudinal envolvendo crianças pré-leitoras inglesas, Rego (1991) testou essa hipótese. A autora demonstrou uma relação causal entre as tarefas de consciência sintática e a leitura contextual. Já a consciência fonológica não contribuiu para leitura no contexto, apenas para tarefas de leitura de palavras simples. Entre as medidas utilizadas por Rego estava a de completar sentenças. Nessa tarefa uma palavra é omitida e a criança tem que utilizar seu conhecimento gramatical para completá-la. Como essa é uma tarefa de consciência sintática, esperava-se que ela estivesse associada à leitura contextual e não à leitura de palavras isoladas. No entanto, os escores nessa tarefa também contribuíram para a leitura de pseudopalavras e palavras isoladas. Esses resultados apontam para uma maior influência da consciência sintática para alfabetização do que a inicialmente sugerida pelos autores.

Rego (1995) investigou a contribuição da consciência sintática para a leitura de palavras em isolamento e no contexto entre crianças brasileiras. Em um estudo longitudinal com crianças pré-leitoras, Rego mediu a habilidade das crianças corrigirem palavras desordenadas numa frase (tarefa de consciência sintática). Cerca de um ano mais tarde a autora mediu a habilidade de as crianças lerem pseudopalavras, leituras isoladas, palavras inseridas num contexto e compreensão de texto. Se a consciência sintática contribui para decodificação na leitura, então os escores na tarefa de consciência sintática devem predizer os escores na tarefa de decodificação da leitura (leitura de pseudopalavras).

Os resultados das correlações feitas entre as diversas medidas tomadas por Rego mostram que a medida de consciência sintática se correlacionou de forma estatisticamente significativa e positiva com a tarefa de facilitação contextual, mas não com a tarefa de leitura de palavras isoladas ou de palavras inventadas. $O$ resultado da regressão múltipla que avaliou a contribuição dos escores na tarefa de consciência sintática para a leitura contextual, um ano depois, mostrou um resultado estatisticamente significativo, mesmo após controlar a idade e os escores na tarefa de memória de trabalho. A autora concluiu que no caso do português, e provavelmente de outras ortografias regulares, as pistas sintático-semânticas não ajudam no entendimento das regras de correspondência entre letra e som, mas sim na utilização de pistas contextuais na leitura.

Guimarães (2003) investigou a relação entre consciência sintática e consciência fonológica e o desenvolvimento da leitura e escrita em crianças com dificuldades de aprendizagem cursando a terceira e a quarta séries do ensino fundamental. A autora não achou diferença entre a performance de crianças com dificuldades de alfabetização e os controles de mesma habilidade de escrita nas mais jovens em idade. No entanto, as crianças de terceira e quarta séries sem dificuldades de leitura tiveram desempenho superior ao das crianças com dificuldade e ao das mais jovens. Esses resultados sugerem que embora não haja um déficit específico da leitura das crianças com dificuldades de aprendizagem, no que diz respeito à consciência sintática, a escolarização pode ter papel no desenvolvimento dessa habilidade.

Entender como a consciência morfossintática se desenvolve e como ela interage com a alfabetização se torna fundamental para que possamos pensar em estudos de intervenção e práticas pedagógicas que facilitam a aquisição da leitura e da escrita. Este estudo foi delineado para investigar o desenvolvimento da consciência morfossintática. Estamos interessados em saber se a habilidade de refletir sobre os aspectos sintáticos da língua interage de fato com o processo de alfabetização. Comparando a performance de crianças de duas séries escolares em processo de alfabetização, podemos observar se a experiência com a alfabetização afeta o desempenho nas tarefas de consciência sintática.

Uma segunda pergunta feita é se há diferenças no desenvolvimento da consciência morfossintática em diferentes contextos culturais em que o desenvolvimento ocorre. Raz e Bryant (1989) mostram que crianças de baixa renda inglesa apresentam o mesmo nível de desenvolvimento da consciência fonológica que crianças de classe média; contudo, assim que a escolarização se inicia o desenvolvimento começa a mudar. As crianças de classe média apresentam desempenho superior. Soares e Cardoso-Martins (1989) mostraram que em escolas de classe média há um maior número de atividades que desenvolvem habilidades de consciência fonológica do que em escolas públicas.

Outros trabalhos têm mostrado que há diferenças no desenvolvimento linguísticos de crianças de acordo com o ambiente em que vivem (Olson, 1988) e que atividades realizadas em casa 
podem afetar o desenvolvimento de habilidades que afetam o desempenho escolar (ver Papalia \& Olds, 2000, para uma revisão). No caso do estudo de Olson, ele se referia a diferentes classes sociais. No segundo caso, refere-se a atividades de linguagem realizadas pelos pais com as crianças em casa

Se os contextos do desenvolvimento afetam o desempenho em tarefas de consciência sintática, esperamos encontrar diferenças na performance das crianças nesses dois tipos de atividade quando diferentes ambientes são comparados. Esses resultados podem nos oferecer subsídios para intervenções e modificações na prática pedagógica para melhorar o desempenho escolar de crianças em diversos contextos educativos. Não está no escopo deste estudo estabelecer quais as diferenças ambientais que afetam o desenvolvimento morfossintático, apenas explorar essa questão para observar sua pertinência como hipótese para estudos futuros.

Mota (1996) fez uma revisão dos estudos que investigaram o desenvolvimento da consciência sintática. Os resultados sugerem uma gradação no nível de consciência sintática decorrente do grau de controle cognitivo necessário para realizar as tarefas metalinguísticas. Os resultados obtidos pela autora foram semelhantes aos relatados por uma revisão mais recente da literatura feita por Correa (2004).

Mota (1996) mostra que o julgamento de sentenças parece ser a tarefa mais fácil na bateria de tarefas que avalia a consciência sintática. Nessa tarefa a criança tem que dizer se uma sentença é gramaticalmente correta ou não. Repare que a demanda cognitiva da tarefa é reduzida, pois a criança não precisa manipulá-la, apenas decidir se a sentença é correta ou não.

Hakes (1980) chama atenção para o fato de que muitas crianças que acertam a tarefa de julgamento, quando solicitadas a explicar sua resposta oferecem justificativas que demonstram um julgamento semântico e não sintático. Um outro aspecto metodológico importante no que diz respeito à tarefa de julgamento de sentenças é que o julgar sentenças corretas é mais fácil do que julgar sentenças incorretas. $\mathrm{Na}$ verdade, há uma tendência a aceitar todas as sentenças na frase quando os sujeitos não são capazes de fazer julgamentos metalinguísticos (Gombert, 1992). Portanto, é possível que haja diferenças no grau de reflexão metalinguística envolvida na realização da tarefa de julgamento de sentenças corretas e incorretas.
Dois outros testes, o de identificação do erro e o de correção, parecem requerer um controle cognitivo maior para sua realização do que a tarefa de julgamento. Nesses testes as crianças têm que identificar o erro, no primeiro caso, e depois, no segundo, identificar o erro e buscar a resposta correta para consertar a sentença. Embora esses dois testes requeiram graus de controle cognitivo maiores do que o de julgar sentenças, Gombert (1992) argumenta que eles ainda são muito influenciados pela semântica e pelo conhecimento tácito da língua, portanto, podem exigir um grau menor de consciência sintática.

Mota, MacLean e Gombert (1995) adaptando as tarefas de julgamento, correção e a de Gombert para o português, obtiveram resultados empíricos que reforçam a ideia de uma progressão na consciência sintática. Os autores deram tarefas de julgamento, identificação e correção a adultos e crianças de primeira série a quarta série do ensino fundamental. Os resultados mostram que para todas as séries a tarefa de julgamento foi mais fácil do que as tarefas de correção e que a performance em todas as tarefas melhorava com a escolarização.

O terceiro ponto investigado são possíveis diferenças de performance que podem ser encontradas entre três tarefas de consciência sintática: julgamento de sentenças corretas, julgamento de sentenças incorretas e correção. Os resultados são discutidos quanto ao grau de controle metalinguístico observado.

\section{Método}

\section{Amostra}

Cinquenta e quatro crianças participaram desse estudo: vinte e seis crianças de primeira série (média de idade 80 meses, dp 6,5), 13 de escolas particulares e 13 de escolas públicas; vinte e oito crianças de segunda-série (média de idade 90 meses, dp 7,1), dessas 16 eram de escolas particulares e 12 de escolas públicas. Todas as escolas eram da região urbana de uma cidade de porte médio de Minas Gerais. As escolas públicas atendem em geral crianças de baixa renda e as escolas particulares, crianças de classe alta e média.

\section{Procedimento}

As crianças foram entrevistadas individualmente na escola. $\mathrm{Na}$ primeira entrevista foram avaliadas consciência fonológica e desempenho escolar. $\mathrm{Na}$ segunda, as medidas de 
consciência sintática. $\mathrm{Na}$ terceira, medidas de desempenho cognitivo foram tomadas. As medidas de consciência fonológica não serão discutidas aqui.

\section{Instrumentos}

\section{Tarefas de consciência sintática}

A) Morfologia produtiva (baseada no teste de Berko, 1958): nesse teste os participantes tiveram que completar sentenças flexionando pseudopalavras. Por exemplo: "Este é um Zugue. Aqui estão dois?"

B) Julgamento e correção: nessa tarefa os participantes tiveram que julgar a gramaticalidade das sentenças corretas; julgar a gramaticalidade das sentenças incorretas e corrigir o erro.

\section{Leitura e ditado}

O teste de leitura foi composto de palavras frequentes e não frequentes da língua portuguesa. As palavras foram retiradas das listas de palavras frequentes e não frequentes de Pinheiro \& Keys (1987, apud Cardoso-Martins, 1997).
Avaliação cognitiva

Um subconjunto do ITPA (Ilinois Test of Psycholinguistic Abilities) que avalia memória de trabalho foi aplicado.

\section{Resultados}

A Tabela 1 mostra a média e o desvio padrão para o número de respostas corretas para cada teste de consciência sintática. Os resultados da análise de variância fatorial $(3 \times 2 \times 2)$, com tipo de tarefa como fator de medidas repetidas (três tarefas de consciência sintática), escola (particular e pública) e série (primeira e segunda) como fatores entre sujeitos e o número de respostas corretas para as três tarefas de consciência como variável dependente mostraram um efeito significativo para tipo de tarefa $\left(F_{(1,50)}=6,75 ; p<0,01\right)$. O resultado do Teste $t$ usado para comparações post hoc mostrou que a tarefa de julgamento de sentenças corretas apresentou desempenhos melhores do que as tarefas de sentenças incorretas $\left(t_{(53)}=5,1 ; p<0,001\right)$ e de correção de sentenças $\left(t_{(53)}=-2,58 ; p<0,01\right)$. Diferenças significativas também foram encontradas entre as tarefas de julgamento de sentenças incorretas e a tarefa de correção $\left(t_{(53)}=3,46 ; p<0,001\right)$. Este resultado não foi $\mathrm{o}$ predito pela hipótese. A tarefa de correção foi mais fácil para as crianças.

Tabela 1. Média e desvio padrão para respostas corretas nas tarefas de consciência sintática (total 6 sentenças)

\begin{tabular}{llccc}
\hline Série & Escola & $\begin{array}{c}\text { Julgamento de } \\
\text { sentenças corretas }\end{array}$ & $\begin{array}{c}\text { Julgamento de sentenças } \\
\text { incorretas }\end{array}$ & Correção \\
\hline C.A & Particular & $5,00(1,07)$ & $4,87(1,13)$ & $5,25(0,76)$ \\
& Pública & $4,77(1,92)$ & $3,38(1,56)$ & $3,77(1,74)$ \\
$1^{a}$ série & Particular & $5,92(0,28)$ & $4,54(1,61)$ & $5,35(2,37)$ \\
& Pública & $5,08(1,31)$ & $3,17(1,70)$ & $4,00(1,46)$ \\
\hline
\end{tabular}

Não houve resultados estatisticamente significativos para diferenças entre as séries $\left(F_{(1,50)}=0,32 ; p>0,57\right)$. A interação tipo de tarefa $e$ séries não foi significativa $\left(F_{(1,50)}=0,75 ; p>0,39\right)$. No entanto, houve um efeito significativo para escola $\left(F_{(1,50)}=9,8 ; p<0,003\right)$. As crianças de escola particular tiveram performance superior às da escola pública. As interações tipo de tarefa $x$ escola $\left(F_{(1,50)}=2,18 ; p>0,14\right)$, série $x$ escola $\left(F_{(1,50)}=0,034\right.$; $p>0,85)$ e tipo de tarefa $\times$ escola $\times$ série $\left(\mathrm{F}_{(1,50)}=0,05\right.$; $p>0,82)$ não foram significativas.

Para testar se a performance nas tarefas de consciência sintática eram associadas à leitura e escrita, correlações de Pearson foram realizadas. Os resultados mostram que as tarefas de consciência sintática correlacionaram-se de forma significativa e positiva com as tarefas de leitura e escrita. A Tabela 2 mostra o resumo desses resultados.

\section{Discussão}

A primeira pergunta que fizemos foi se a consciência sintática interage com a alfabetização. A princípio nossos resultados não dão suporte a essa idéia, uma vez que não encontramos diferenças significativas entre série e nenhuma das interações envolvendo as séries escolares, escola, e o tipo de tarefa.

Contudo, uma associação entre a escrita e consciência sintática foi encontrada. Correlações 
significativas e positivas foram encontradas entre a performance das crianças no teste de leitura e escrita e todas as tarefas de consciência sintática. Esses resultados mostram que as crianças com os melhores desempenhos no teste de leitura e escrita tiveram melhores performances nos testes de consciência sintática. Correlações não estabelecem relações de causa e efeito, porém indicam que a consciência sintática interage com a aquisição da escrita.

Tabela 2. Coeficientes de Correlação de Pearson para tarefas de consciência sintática, leitura e escrita.

\begin{tabular}{lcccc}
\hline & 2 & 3 & 4 & 5 \\
\hline 1. correção & $* *, 36$ & $*, 29$ & $* *, 51$ & $* *, 45$ \\
2. Julg. Sentenças Corretas & &,- 12 & $*, 34$ & $* *, 35$ \\
3. Julg. Sentenças incorretas & & & $* *, 39$ & $* *, 35$ \\
4. leitura & & & & $* *, 88$ \\
5. escrita & & & \\
\hline
\end{tabular}

$* * p<0,01 ; * p<0,05$

Então, como interpretar os resultados? É possível que o conhecimento das crianças sobre a língua escrita não tenha sido marcante o suficiente entre a primeira e a segunda série em decorrência de diferenças no tipo de ensino adotado pelas diferentes escolas. Como nas correlações os dados foram agrupados, a análise se concentrou na variação entre os sujeitos. Outros estudos que garantam a homogeneidade dos grupos quanto ao ensino se fazem necessário para responder definitivamente nossa primeira pergunta.

A segunda questão levantada diz respeito aos contextos do desenvolvimento. As crianças de escola particular obtiveram performance superior à das crianças de escola pública nas tarefas de consciência sintática. Esse resultado não pode ser explicado simplesmente por uma maior experiência das crianças de escola particular com a língua escrita, pois não houve diferenças entre as séries ou interações com as séries escolares significativas. As crianças com mais experiência escolar não tiraram vantagem dessa experiência na realização das tarefas em relação às mais novas. Diferenças em desempenho escolar têm sido encontradas entre crianças de escolas públicas e particulares no Brasil. O índice de crianças com dificuldades em se alfabetizar é maior nas escolas públicas, podendo chegar a $50 \%$ das crianças matriculadas (Scoz, 1998). No entanto, a falta de efeito de série escolar, encontrada neste estudo, indica que a questão dos diferentes contextos de ensino é mais qualitativa do que quantitativa.

Esses resultados podem sugerir que experiências vividas pelas crianças nos diferentes contextos culturais em que participam podem levar a diferenças no desenvolvimento de habilidades linguísticas. Olson (1988), por exemplo, argumenta que há diferenças de experiência com a linguagem entre crianças de diferentes classes sociais. Embora essas diferenças de experiência não afetem o desenvolvimento linguístico geral, uma área afetada é o desenvolvimento metalinguístico. Para o autor, as interações das crianças com a mãe podem ser responsáveis pelo desenvolvimento das habilidades metalinguísticas. Mães que expandem a linguagem das crianças estão ajudando a desenvolver a habilidade de refletir sobre ela. Olson (1988) observou que as mães de classe média expandem a linguagem com mais frequência do que as de classe baixa.

Estudos futuros devem estudar essa hipótese para tentar esclarecer esta questão. No Brasil as escolas públicas atendem a uma clientela, em geral, de classe baixa, enquanto as escolas privadas atendem a uma clientela de classe média e alta. Seria interessante verificar se há diferenças de interação entre mãe e criança nas diferentes classes sociais no Brasil, no que diz respeito ao desenvolvimento da linguagem e o efeito dessa interação sobre o desenvolvimento metalinguístico.

Porém, essa não é a única explicação possível para os resultados observados. Os resultados encontrados por Raz e Bryant (1990), citados na introdução deste estudo, sugerem que as crianças de classe média são mais eficientes em usar as experiências escolares para desenvolver habilidades metalinguísticas. Por outro lado, Cardoso-Martins e Soares (1989), mostram que há diferenças no número de atividades que desenvolvem habilidades metalinguísticas em diferentes contextos escolares. No estudo de Cardoso-Martins e Soares (1989) as escolas particulares desenvolviam mais atividades que melhoram as habilidades metalinguísticas. Assim, uma outra hipótese que deve ser investigada são possíveis diferenças entre práticas pedagógicas 
entre escolas públicas e particulares em relação ao desenvolvimento da linguagem oral e escrita. Por fim, as crianças de escolas particulares tendem a ingressar na escola antes das de escola pública, na educação infantil. Esses anos de escolarização podem fazer diferença no desenvolvimento de habilidades metalinguísticas.

Nosso estudo, embora levante mais questões do que apresente respostas, mostra a importância de se ampliar as investigações nessa área do desenvolvimento metalinguístico, especialmente da consciência morfossintática, ainda pouco estudada. Esta é uma questão complexa, cujo esclarecimento é fundamental para que possamos pensar quanto à prevenção e promoção dos problemas de aprendizagem.

No que se refere ao diferente grau de habilidade metalinguística que requer as diferentes tarefas de consciência sintática, os resultados estão de acordo com a literatura que considera que a tarefa de julgamento de sentenças corretas é mais fácil do que o de sentenças incorretas e correção. O julgamento de sentenças corretas é mais próximo do processamento da linguagem, que é automático, por isso de menor demanda cognitiva.

Esperava-se que a tarefa de julgamento de sentenças incorretas fosse mais fácil do que a tarefa de corrigir sentenças, mas os dados foram opostos aos esperados. A tarefa de julgar sentenças foi mais difícil do que a de correção. A tarefa de julgamento foi feita antes da de correção. Como as sentenças nas duas tarefas eram as mesmas, para garantir que a dificuldade da tarefa se mantinha entre tarefas, nosso resultado pode ser consequência de um efeito da aprendizagem das crianças. Porém, esses resultados mostram que a maior dificuldade encontrada pelas crianças é estabelecer o erro, julgamento que parece ser feito com base na escolha correta das sentenças. Em outras palavras, as crianças conseguem corrigir as sentenças que julgam incorretas, embora essa ação envolva a busca da resposta correta no léxico.

\section{Referências}

Barrera, S. \& Maluf, M. R. (2003). Consciência metalinguística e alfabetização: um estudo com crianças da primeira série do ensino fundamental. Psicologia: Reflexão e Crítica, 16 (3), pp. 491-502.

Bialystok, E. (1993). Metalinguistic awareness: The development of children's representations of language. Em C. Pratt \& A.F. Garton (Orgs.).
Systems of representation in children: development and use (pp. 211-234). Chichester: Wiley.

Bowey, J. (2005). Grammatical sensitivity: its origins and potential contribution to early reading skill. Journal of Experimental Child Psychology, 90, 318-343.

Bradley, L. \& Bryant, P. (1985). Children's reading problem. Oxford: Basil Blackwells.

Capovilla, A. \& Capovilla, F. (2000). Efeitos do treino de consciência fonológica em crianças com baixo nível sócio-econômico. Psicologia Reflexão e Crítica,13 (1), 7-24.

Cardoso-Martins, C. (1995). Consciência fonológica e alfabetização. Petrópolis: Vozes.

Cardoso-Martins, C. (1997). A sensibilidade e rima e ao fonema $e$ a aquisição da leitura em crianças normais e indivíduos com a sindrome de Down: um estudo correlacional. Tese para obtenção de título de professor titular. Minas Gerais: Universidade Federal de Minas Gerais..

Correa, J. (2004). A avaliação da consciência sintática na criança. Psicologia: Teoria e Pesquisa, 20, (1), 69-75.

Correa, J. (2005). A avaliação da consciência morfossintática na criança. Psicologia: Reflexão e Crítica, 18 (1), 91-97.

Garton, A. \& Pratt, C. (1989). Learning to be literate. The development of Spoken \& Written Language. Oxford: Basil Blackwell.

Gombert J. E. (1992). Metalinguistic Development. Hertfordshire: Harverster Wheatsheaf.

Gombert, J. E. (2003). Atividades metalinguísticas e aquisição da leitura. Em M. R. Maluf (org.). Metalinguagem e aquisição da escrita (pp. 19-64). São Paulo: Casa do Psicólogo.

Goswami, U. \& Bryant, P. (1990). Phonological skills and learning to read. East Sussex, UK: Lawrence Erlbaum.

Guimarães, S. R. (2003). Dificuldades no desenvolvimento da lecto-escrita: o papel das habilidades metalinguísticas. Psicologia: Teoria e Pesquisa, 19(1), 33-45.

Hakes D. (1980). The development of metalinguistic abilities in children. Berlim: Springer-Verlag.

Mota, M. (1996). Children's role of grammatical rules in spelling. Oxford University: Experimental Psychology Department. 
Nunes, T. \& Bryant, P. (2006). Improving literacy by teaching morphemes. London: Rotdlege.

Olson, D. R. (1988). See jumping! Some oral language antecedents of literacy. Em N. Mercer (org.). Language and Literacy from an educational perspective. Milton Keynes: Open University Press.

Papalia, D. \& Olds, S. (2000). Desenvolvimento Humano. Porto Alegre: ARTMED.

Plaza, M. \& Cohen, H. (2003). The interaction between phonological processing, syntactic awareness, and naming speed in the reading and spelling performance of first-grade children. Brain and Cognition, 53, 257-292.

Plaza, M. \& Cohen, H. (2004). Predictive influence of phonological processing, morphological/syntactic skill, and naming speed on spelling performance. Brain and Cognition, 55, 368-373.

Raz, I. S. \& Bryant, P. (1990). Social background, phonological awareness and children's reading. British Journal of Developmental Psychology, 8, 209-225.

Rego, L. (1991). The role of early linguistic awareness in children's reading and spelling. Oxford
University: Experimental Psychology Department.

Rego, L. (1995). Diferenças individuais na aprendizagem inicial da leitura: papel desempenhado por fatores metalinguísticos. Psicologia: Teoria e Pesquisa,11(1), 51-60.

Rego, L. \& Bryant, P. (1993). The connections between phonological, syntactic and semantic skills and children's reading and spelling. European Journal of Psychology, 3, 235-246.

Scoz, B. (1998). Psicopedagogia e realidade escolar. Petrópolis: Vozes.

Soares, M. \& Cardoso-Martins, C. (1989). A consciência fonológica de crianças de classes populares: o papel da escola. Revista Brasileira de Estudos Pedagógicos, 70 (164), 86-97.

Tunmer, W. (1990). The role of language prediction skills in beginning reading. New Zealand Journal of Educational Studies, 25(2), 95112.

Recebido em abril de 2008

Reformulado em outubro de 2008 Aprovado em janeiro de 2009

Sobre os autores:

Márcia da Mota é professora adjunta da Universidade Federal de Juiz de Fora, doutora em Psicologia pela Universidade de Oxford - Inglaterra, coordenadora do Programa de Pós-Graduação em Psicologia da UFJF, líder do Grupo de Pesquisa em Psicologia do Desenvolvimento e Relações Interpessoais.

Daniela Mota é aluna de graduação e bolsista de Iniciação Científica da Universidade Federal de Juiz de Fora.

Junia Cota é aluna de graduação e bolsista de Iniciação Científica da Universidade Federal de Juiz de Fora.

Stella Mansur é aluna de graduação e bolsista de Iniciação Científica da Universidade Federal de Juiz de Fora.

Simone Lima é graduada em Psicologia, aluna do Curso de Mestrado do Programa de Pós-Graduação em Saúde Brasileira da Universidade Federal de Juiz de Fora.

Átila Calzavara é aluno de graduação e bolsista de Iniciação Científica da Universidade Federal de Juiz de Fora.

Natália Cunha é aluna de graduação e bolsista de Iniciação Científica da Universidade Federal de Juiz de Fora.

Eliane Banhato é mestre em Psicologia do Desenvolvimento pela UFMG e aluna do Curso de Doutorado do Programa de Pós-graduação em Saúde Brasileira da Universidade Federal de Juiz de Fora. 